\title{
All-optically induced quasi phase matching in SiN waveguides for second harmonic generation enhancement
}

\author{
Davide Grassani ${ }^{1}$, Adrien Billat ${ }^{1}$, Martin H. P. Pfeiffer ${ }^{2}$, Svyatoslav Kharitonov ${ }^{1}$, Tobias J. Kippenberg ${ }^{2}$, \\ Camille-Sophie Brès ${ }^{1 *}$ \\ ${ }^{1}$ Ecole Polytechnique Fédérale de Lausanne (EPFL), Photonic Systems Laboratory, CH-1015, Switzerland \\ ${ }^{2}$ Ecole Polytechnique Fédérale de Lausanne (EPFL), Laboratory of Photonics and Quantum Measurements, CH-1015, Switzerland \\ Author e-mail address: camille.bres@epfl.ch
}

\begin{abstract}
We report more than 30dB second harmonic generation enhancement in SiN waveguide by all-optical writing of a persistent $\chi^{(2)}$ grating. Phase matching peaks are observed for different writing wavelengths.
\end{abstract}

OCIS codes: (190.4390) Nonlinear optics, integrated optics; (190.2620) Harmonic generation and mixing.

\section{Introduction}

Thanks to tight light confinement and fabrication flexibility, CMOS compatible materials like silicon (Si) and silicon nitride ( $\mathrm{SiN}$ ) allowed the integration of various $\chi^{(3)}$-based optical functionalities on chip [1][2]. Integration of $\chi^{(2)}$-based effects would enable even more efficient devices and new compelling applications like on-chip detection of telecom signal with integrated silicon detectors [3], photon pair generation with easier pump filtering [4], and self-referencing of frequency comb [5]. Unfortunately, $\chi^{(2)}$ theoretically vanishes in the dipole approximation both for $\mathrm{Si}$ and $\mathrm{SiN}$. On the one hand, attempts were reported to integrate well performing $\chi^{(2)}$ materials, such as lithium niobate [6], on CMOS waveguides. On the other hand, photonic confinement leading to resonant structures with limited bandwidth [7], or symmetry breaking in strained Si waveguides [8] were employed to enhance the efficiency of second order processes. $\mathrm{SiN}$, in particular, is a very promising material as it features a very a large transparency window (from visible to mid-infrared), and already exhibited interesting bulk second order nonlinearity [9]. However, the conversion efficiency is often limited by the modal phase matching condition, which imposes stringent constraint on the waveguide design. Here we show the enhancement by more than $30 \mathrm{~dB}$ of the second harmonic of a narrow linewidth telecom pulsed laser. We observe a similar SHG enhancement changing the pump laser wavelength within the erbium window. Finally, probing the waveguide in continuous-wave (CW), the SHG peak shifts accordingly to the employed pump wavelength. We report a maximum SHG efficiency of $1.23 \cdot 10^{-6} \mathrm{~W}^{-1} \mathrm{~cm}^{-1}$ and we estimate $\chi^{(2)}$ to be about $0.3 \mathrm{pm} / \mathrm{V}$.

\section{Results and discussion}
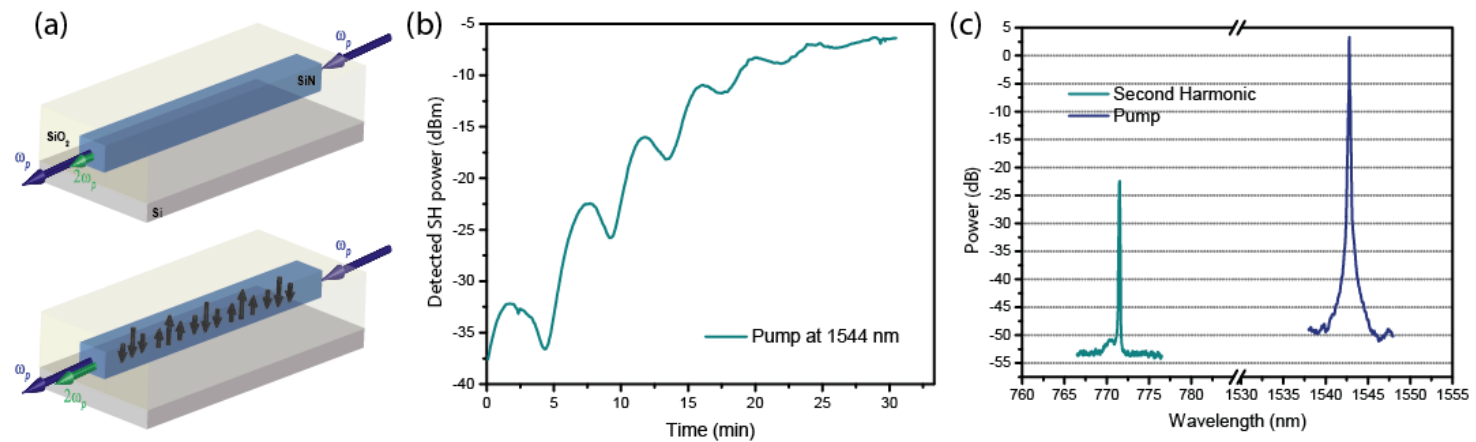

Fig. 1: (a) Schematic principle of $\chi^{(2)}$ grating inscription in SiN waveguides, where $\omega_{\mathrm{p}}$ is the pump frequency. (b) SH power growth over time, during the grating inscription, when $90 \mathrm{~W}$ peak power at $1544 \mathrm{~nm}$ are coupled into the waveguide (c) Pump and harmonic spectra obtained after growth, for a coupled pump peak power of $90 \mathrm{~W}$.

The waveguide is made of stoichiometric $\mathrm{SiN}$ buried in $\mathrm{SiO}_{2}$ (see Fig. 1(a)) and is fabricated according to the photonic Damascene process [10], featuring low attenuation $(0.2 \mathrm{~dB} / \mathrm{cm})$. It is $4 \mathrm{~cm}$ long with a cross section of $0.87 \times 1.5 \mu \mathrm{m}$ and terminated by inverse taper mode converters. The pump consists of a modulated tunable C-band laser, amplified by a cascade of erbium-doped fiber amplifiers. The resulting pulses are 200 ps long, at a repetition rate of $25 \mathrm{MHz}$. At the output facet, short and long pass filters are used to remove the remaining pump and thirdharmonic green light, before sending the output to a Si power detector. Fig. 1(b) shows the signal collected on the detector, found to be TM polarized, when a pump peak power of $90 \mathrm{~W}$ at $1544 \mathrm{~nm}$ pump is coupled into the 
waveguide on the TM fundamental mode. The initially weak SH grows by $30 \mathrm{~dB}$ over several tens of minutes, while the coupled pump power remains constant, to reach a conversion level of $1.23 \cdot 10^{-6} \mathrm{~W}^{-1} \mathrm{~cm}^{-1}$. Fig. 1(c) features a spectrum showing the pump and corresponding second harmonic signals. Subsequently, we probe the waveguide with a CW laser, swept over the C-band. We observe a SH power peak centered at the pumping wavelength. Changing the pump wavelength, we observe a similar enhancement to that reported in Fig. 1(b), while the SHG peak wavelength changes accordingly when probing the waveguide in CW. These probing results are shown in Fig. 2(a),

The SHG enhancement, with a clear phase matching peak at the pump wavelength, has been observed in silica fibers pumped by a $\mathrm{kW}$-level pulsed Nd:YAG laser [11]. The effect is attributed to a periodic modification of the material $\chi^{(2)}$ that progressively builds up during the illumination due to a coherent photogalvanic effect caused by the pump and SH waves [12]. The period of the resulting $\chi^{(2)}$ grating is twice the coherence length between the pump and SH waves, allowing for quasi phase matched SHG at the pump frequency. From finite element method simulations, we retrieve the waveguide dispersion curves for the pump and SH modes (Fig. 2(b)). We see that a weak and poorly phase matched SHG is expected to take place initially on the $8^{\text {th }}$ TM mode at our pump frequency. Thanks to the experimental parameters and to the simulated pump and second harmonic mode profiles, we retrieve a $\chi^{(2)}$ of $0.3 \mathrm{pm} / \mathrm{V}$ by fitting the data from Fig. 2(c). The latter plots the generated SH power as a function of coupled probe power, while the fitting model assumes quasi phase matching.
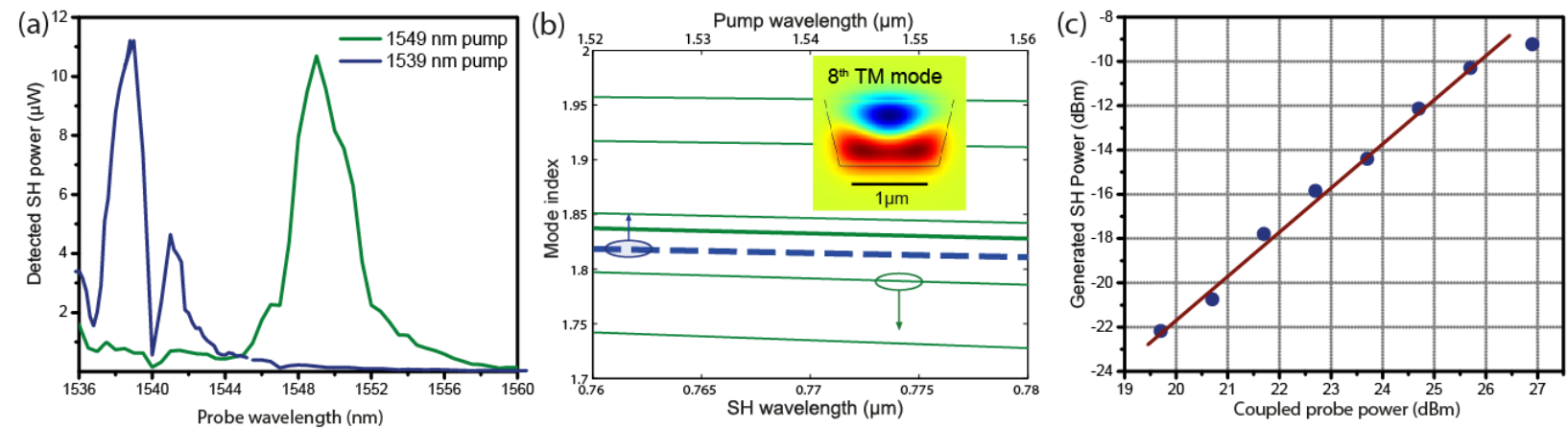

Fig. 2: (a) $\mathrm{SH}$ power as a function of the $\mathrm{CW}$ probe wavelength, in waveguides previously pumped at 1539 and $1549 \mathrm{~nm}$. Phase-matching peaks are observed around each pump wavelength. (b) Index matching curves for the fundamental TM mode at the pump wavelengths (blue dashed line), and for the higher order TM modes at the SH wavelengths (green lines). The inset represents the simulated second harmonic mode profile. (c) SH power (estimated in the waveguide) as a function of the coupled $\mathrm{CW}$ probe power, for a $1543 \mathrm{~nm}$ probe in a waveguide previously pumped at $1543 \mathrm{~nm}$. Dots are experimental data while the red line is a guide for the eye representing the expected slope of 2 for SHG.

This result represents a straightforward way to increase SHG efficiency in a CMOS compatible material like SiN for arbitrary wavelengths in the telecom band. At quasi-phase matching, we report a SHG efficiency comparable to that reported in SiN resonant structures [7], with the benefit of a wider bandwidth. Even higher conversion efficiencies are expected using Si-rich SiN, which exhibits $\chi^{(2)}$ values ten times larger than the stoichiometric compound [13]. Phase matched SHG in SiN waveguides can then compete with conventional $\chi^{(2)}$ materials, but with fairly easier onchip integration, and with the capability of dynamically and all-optically reconfigure the phase matching configuration.

This work was supported by the European Research Council under grant agreement ERC-2012-StG 306630-MATISSE, and by Contract HR0011-15-C-0055 from the Defense Advanced Research Projects Agency (DARPA), Defense Sciences Office (DSO).

[1] X. Liu, et al. "Mid-infrared optical parametric amplifier using silicon nanophotonic waveguides," Nat. Photonics 4, 557 (2010).

[2] J. S. Levy, et al. "CMOS-compatible multiple-wavelength oscillator for on-chip optical interconnects," Nat. Photonics 4, 37 (2010).

[3] M.-J. Lee and W.-Y. Choi, "A silicon avalanche photodetector fabricated with standard CMOS technology with over 1 THz gain-bandwidth product," Opt. Express 18, 24189 (2010).

[4] N. C. Harris, et al. "Integrated source of spectrally filtered correlated photons for large-scale quantum photonic systems," Phys. Rev. X 4, 110 (2014).

[5] S. Miller, et al. "On-chip frequency comb generation at visible wavelengths via simultaneous second- and third-order optical nonlinearities," Opt. Express 22, 26517 (2014).

[6] L. Chang, et al. "Thin film wavelength converters for photonic integrated circuits," Optica 3, 531-535 (2016).

[7] J. S. Levy, et al," "Harmonic generation in silicon nitride ring resonators," Opt. Express 19, 11415 (2011).

[8] M. Cazzanelli, et al. "Second-harmonic generation in silicon waveguides strained by silicon nitride," Nat. Mater. 11, 148-154 (2011).

[9] M. W. Puckett, et al. "Observation of second-harmonic generation in silicon nitride waveguides through bulk nonlinearities" Opt. Express 24, 16923 (2016).

[10] M.H.P. Pfeiffer et al. "Photonic Damascene process for integrated high-Q microresonator based nonlinear photonics," Optica 3, 20 (2016).

[11] M. C. Farries, et al. "Second-harmonic generation in an optical fibre by self-written x(2) grating," Electronics Letters 23, 322 (1987).

[12] D. Z. Anderson, et al. "Model for second-harmonic generation in glass optical fibers based on Asymmetric Photoelectron Emission From Defect Sites," Opt. Lett. 16, 796 (1991).

[13] A. Kitao, et al. "An investigation into second harmonic generation by Si-rich SiN x thin films deposited by RF sputtering over a wide range of Si concentrations," J. Phys. D. Appl. Phys. 47, 215101 (2014). 